\section{Moderner Neubau}

Nach einer verheerenden Brandstiftung im März 2013 konnte nun ein Neubau mit innovativen Erweiterungsmöglichkeiten vorgestellt werden. „Wir sind auferstanden, wie der legendäre Phönix aus der Asche. Das war nach dem schlimmen Brandschaden durch die Brandstiftung eines Einzeltäters und ihre betriebswirtschaftlichen Folgen 2013 so zunächst nicht zu erwarten. Allerdings haben beim Brandschadensmanagement und in der Betriebsfortführung alle Mitarbeiter optimal zusammengearbeitet und sich sehr

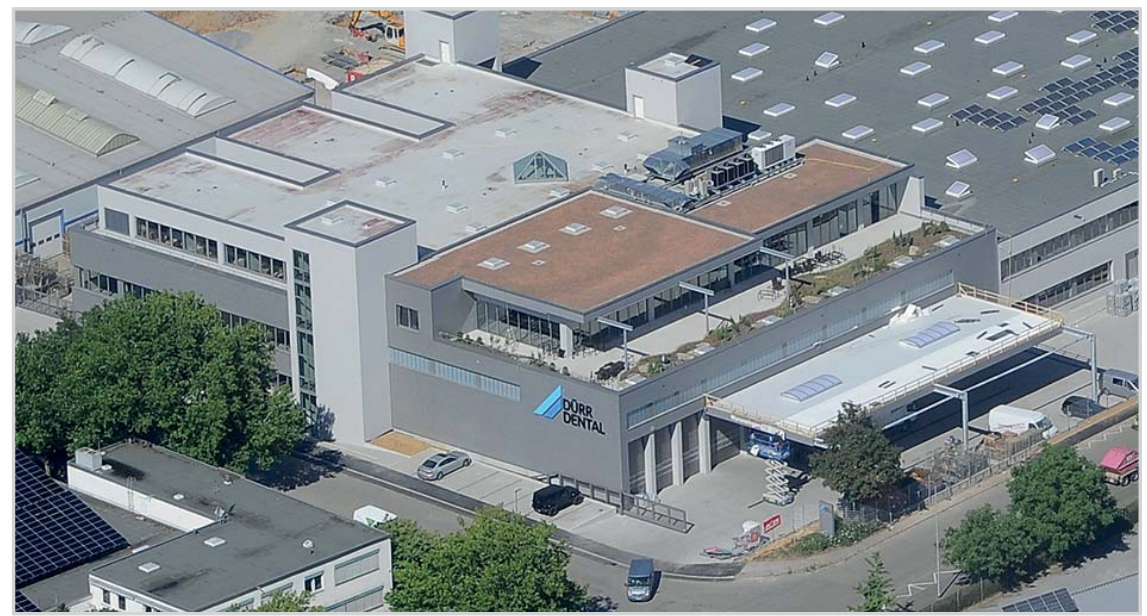

engagiert. Heute sind wir noch stärker als zuvor“, sagt Martin Dürrstein, Vorstandsvorsitzender der Dürr Dental AG.

Der neue Erweiterungsbau demonstriert, wie aus einem unverschuldeten Unglück auch neue Chancen für die Entwicklung eines Unternehmens entstehen können. Das moderne multifunktionale Gebäude mit einer Fläche von $7500 \mathrm{~m}^{2}$ wurde für über 10 Millionen $€$ nach zeitgemäßen Kenntnissen errichtet. Es beinhaltet Teile der Verwaltung, Montage und Logistik,

ein neues Rechenzentrum sowie ein mitarbeiterfreundliches Betriebsrestaurant. Die Investitionen der letzten Jahre am Hauptstandort mit $27000 \mathrm{~m}^{2}$ belaufen sich auf nunmehr über 20 Millionen $€$.

Das Unternehmen wurde 1941 von den Brüdern Karl und Wilhelm Dürr gegründet und ist seit mehreren Jahrzehnten ein weltweit führender Anbieter von dentaler Medizintechnik wie Druckluft- und Absaugtechnologie, digitale Röntgensysteme und Hygieneprodukte für Zahnarztpraxen und Kliniken. Außerdem hat es ein ausgeprägtes Forschungs- und Entwicklungssegment mit über 150 Mitarbeitern. Dies betrifft nicht nur die Entwicklung innovativer Produkte und nachfrageorientierter Dienstleistungen rund um die moderne Zahnheilkunde, sondern auch die Ausbildung von technischen Nachwuchskräften sowie die Schulung und Fortbildung von Zahnärzten und zahnmedizinischem Fachpersonal. Daneben ist es im Inund Ausland sozial engagiert, insbesondere in Afrika durch die regelmäßige Unterstützung des weltweit größten privaten Hospitalschiffes Africa Mercy der internationalen Hilfsorganisation Mercy Ships.

Nach einer Pressemitteilung der Dürr Dental AG, Bietigheim-Bissingen www.duerrdental.com 